\title{
ANTIPSICÓtICOS DE AÇÃO PROLONGADA NO TRATAMENTO DE MANUTENÇÃO DA ESQUIZOFRENIA. PARTE I. FUNDAMENTOS DO SEU DESENVOLVIMENTO, BENEFícIOS E NíVEL DE ACEITAÇÃO EM DIFERENTES PAÍSES E CULTURAS
}

Luiz Paulo de C. Bechelli ${ }^{1}$

Bechelli LPC. Antipsicóticos de ação prolongada no tratamento de manutenção da esquizofrenia. Parte I. Fundamentos do seu desenvolvimento, benefícios e nível de aceitação em diferentes países e culturas. Rev Latino-am Enfermagem 2003 maiojunho; 11(3):341-9.

Neste trabalho são revistos os fundamentos do desenvolvimento dos antipsicóticos de ação prolongada, a aderência ao tratamento, fatores que dificultam o seguimento da prescrição e suas conseqüências, benefícios e aceitação dos antipsicóticos de ação prolongada. A partir dos dados desta primeira parte do trabalho, os seguintes pontos devem ser destacados: a taxa de não aderência ao tratamento na esquizofrenia é em torno de 60\%; por se tratar de uma doença crônica com elevado risco de recaída, a manutenção do medicamento auxilia no seu controle. Os antipsicóticos de ação prolongada foram desenvolvidos exatamente com este objetivo: garantir a administração do medicamento e a sua regularidade, condições essenciais na prevenção da recaída. Apesar dos benefícios inestimáveis do antipsicóticos de ação prolongada, diversos fatores contribuíram para que tivessem uma utilização aquém do seu potencial.

DESCRITORES: antipsicótico, antipsicótico de ação prolongada, antipsicótico depot, esquizofrenia, aderência

\section{LONG-ACTING ANTIPSYCHOTICS IN THE MAINTENANCE TREATMENT OF SCHIZOPHRENIA. PART I. FOUNDATIONS OF ITS DEVELOPMENT, BENEFITS AND ACCEPTANCE LEVEL IN DIFFERENT COUNTRIES AND CULTURES}

In this study, the foundations of long-acting antipsychotics development, the adherence to treatment, the factors that make difficult the following of the prescription and their consequences, the benefits and acceptance of long-acting antipsychotics are revised. Based on the data obtained from the first part of this study, the following issues must be highlighted: the nonadherence treatment level in schizophrenia is about 60\%. Considering schizophrenia a chronic disease with high relapse risk, the maintenance treatment helps its control. The long-acting antipsychotics were developed exactly for the purpose of guaranteeing the administration of the medication and its regularity, which are essential requirements in preventing a relapse. In spite of the immensurable benefits of the long-acting antipsychotics, several factors have contributed to their usage below their potential.

DESCRIPTORS: antipsychotic, long-acting antipsychotic, depot antipsychotic, schizophrenia, adherence

ANTISICÓTICOS DE ACCIÓN PROLONGADA EN EL TRATAMIENTO DE MATENIMIENTO DE LA ESQUIZOFRENIA. PARTE I. FUNDAMENTOS DE SU DESARROLLO, BENEFICIOS Y NIVEL DE ACEPTACIÓN EN DIFERENTES PAÍSES E CULTURAS

En este trabajo revisamos los fundamentos del desarrollo de los antisicóticos de acción prolongada, la adherencia al tratamiento, factores que dificultan el seguimiento de la prescripción y sus consecuencias, así como sus beneficios y aceptación. A partir de los datos de esta primera parte del trabajo, el siguiente punto necesita ser destacado: la tasa de no-adherencia al tratamiento en esquizofrenia está alrededor de 60\%. Por tratarse de una enfermedad crónica, con riesgo elevado de recaída, el mantenimiento del medicamento ayuda en su control. Los antisicóticos de acción prolongada fueron desarrollados exactamente con este objetivo: garantizar la administración del medicamento y su regularidad, condiciones esenciales para prevenir la recaída. A pesar de los beneficios inestimables de los antisicóticos de acción prolongada, diversos factores han contribuido para que en su utilización no se aproveche todo su potencial.

DESCRIPTORES: antisicótico, antisicótico de acción prolongada, antisicótico depot, esquizofrenia, adherencia

\footnotetext{
${ }^{1}$ Psiquiatra, consultório particular, Ribeirão Preto (SP), Assistente Estrangeiro, Université Claude Bernard, Lyon, França, e-mail: bechelli@netsite.com.br
} 
INTRODUÇÃO

Em 1975 começamos a estudar os antipsicóticos de ação prolongada no tratamento da esquizofrenia ${ }^{(1)}$. Realizamos diversos ensaios clínicos na fase de manutenção em ambulatório público ${ }^{(2-3)}$ e em consultório particular $^{(4-5)}$, e na fase de exacerbação aguda com pacientes internados ${ }^{(6-7)}$. Descrevemos o emprego deste tipo de medicamento associado à psicoterapia individual, familiar e de grupo ${ }^{(8)}$, assim como já havíamos destacado anteriormente a colaboração fundamental do serviço social no preparo da alta hospitalar e durante o tratamento em ambulatório público ${ }^{(2,9)}$. Em 1993, publicamos nossa experiência global sobre o assunto ${ }^{(10)}$. Neste momento, 50 anos após a introdução do primeiro antipsicótico, a clorpromazina (amplictil), e 40 anos do primeiro antipsicótico de ação prolongada, o enantato de flufenazina (anatensol depot), além das perspectivas que recentemente nos trouxeram os antipsicóticos de nova geração, passamos a contar também, com o primeiro deste grupo com ação prolongada, risperidona (Risperdal Consta) ${ }^{(11)}$, ampliando os recursos no tratamento da esquizofrenia para manter a constância e estabilidade do emprego do medicamento.

Este trabalho foi preparado a partir de nossos estudos, experiência e contribuição de outros especialistas. É uma atualização do que já havíamos escrito. Nos últimos 10 anos, diversos estudos a duplocego vieram confirmar achados clínicos decorrentes de observações da prática diária ou indicar necessidade de adequações de condutas até então adotadas. Aspectos relacionados à aderência ao tratamento, riscos de recaída e estratégias de tratamento tornaram-se mais precisos.

\section{POR QUÊ ANTIPSICÓTICOS DE AÇÃO PROLONGADA?}

Até o advento dos antipsicóticos, pacientes viviam muitas vezes confinados em sanatórios em decorrência da falta de recurso terapêutico adequado. Na década de 50 , após o impacto e grande entusiasmo diante dos benefícios decorrentes da propagação do uso desses medicamentos no tratamento da esquizofrenia, ocasionando melhora e controle dos sintomas, possibilitando alta hospitalar e retornaro ao convívio social, constatou-se que os doentes, uma vez melhorados, tendiam a tomar irregularmente o medicamento ou até mesmo a interrompê-lo ${ }^{(12)}$. Em conseqüência, recaídas e reinternações passaram a ser comuns. Tornou-se evidente que esses agentes atuavam na fase aguda ou de exacerbação aguda, mudavam intensamente as manifestações e o curso da esquizofrenia, sem, entretanto, curá-la. A partir desta constatação, a estratégia terapêutica preconizada passou a ser a continuidade da medicação para prevenção de novo episódio. Para facilitar o seguimento e a regularidade do tratamento, tanto pelo paciente quanto pelos familiares, procurou-se desenvolver antipsicótico com efeito suficientemente prolongado que permitissem ampliar o intervalo entre as administrações e sem perda da atividade terapêutica ${ }^{(13)}$. Pouco tempo após terem sido feitas estas considerações, foi realizado estudo em animais ${ }^{(14)}$ e apresentada a primeira publicação ${ }^{(15)}$ sobre os resultados clínicos obtidos com o uso do enantato de flufenazina (ação de 15 dias) em 147 doentes. Os resultados destas investigações estimularam o desenvolvimento de outros antipsicóticos de ação prolongada, por exemplo, palmitato de pipotiazina (piportil L4) e haloperidol decanoato (haldol decanoato) com ação de 30 dias $^{(3)}$.

\section{DESENVOLVIMENTO E MECANISMO FARMACOLÓGICO DOS ANTIPSICÓTICOS DE AÇÃO PROLONGADA}

O princípio que norteou o desenvolvimento desses medicamentos foi encontrar uma alternativa que permitisse que a droga ativa tivesse absorção, metabolismo ou eliminação lentos.

Nos denominados depot, o mecanismo de obtenção consiste na esterificação. O éster é um composto formado por reação química entre um álcool e ácido. O álcool é, no nosso caso, um radical da cadeia lateral do antipsicótico e o ácido é representado pelos ácidos graxos. É lenta a difusão da droga do depósito oleoso para o interstício, onde vem a ser hidrolisada progressivamente por enzimas hidrolíticas, encontradas em todos os tecidos, inclusive no sangue e cérebro, liberando a droga ativa ou bioformada. Uma quantidade pode ser transportada do depósito oleoso para depósitos secundários em tecidos gordurosos do organismo.

Em relação à duração do efeito, em regra, quanto mais longa a cadeia do ácido graxo, melhor é a solubilidade 
do éster e menor a difusão a partir do depósito. Assim, observa-se na experiência clínica duração do efeito terapêutico maior para o palmitato, seguido do decanoato e enantato ${ }^{(16)}$. O veículo para a droga esterificada é, em alguns dos depot, o óleo de sésamo que retarda ainda mais a liberação.

Quanto aos derivados do grupo difenilbutilpiperidina - fluspirilene e penfluridol - a ação prolongada é própria da droga e não secundária ao artifício de esterificação.

Quanto à formulação do risperdal consta, foi empregada tecnologia em que microesferas de um polímero contêm a risperidona. Este composto, ao ser diluído em solução aquosa e aplicado i.m., sofre um processo, em que a cadeia do polímero gradualmente se desfaz em ácido glicólico e ácido lático, liberando a risperidona numa taxa constante. Os ácidos glicólico e lático são metabolizados em dióxido de carbono e água e, a seguir, eliminados sem deixar resíduos. A solução aquosa utilizada como veículo torna mais fácil a administração i.m. e ocasiona menor nível de desconforto e dor em relação à solução oleosa.

O mecanismo de liberação da risperidona a partir das microesferas se processa em três estágios. No primeiro ( hidratação), que segue a hidratação e aplicação i.m., com duração aproximada de duas a três semanas, a droga ativa praticamente não é ainda liberada. Na terceira semana (segundo estágio: difusão da droga) é que se inicia de fato a dispersão da risperidona, à medida que as microesferas começam a se desfragmentar. O nível terapêutico é alcançado na quarta semana. Finalmente, sete semanas após a injeção inicial, começa o último estágio (erosão do polímero), com rápida queda do nível plasmático e degradação total da microesfera.

\section{ADERÊNCIA AO TRATAMENTO}

Por esse termo considera-se o comportamento do paciente na aplicação correta da orientação médica, no que se refere ao medicamento, dieta, modificação de hábitos ou comparecimento às consultas ${ }^{(17)}$. A concordância e cooperação no emprego dos procedimentos prescritos tornam-se um problema, em qualquer que seja a doença, quando é o próprio paciente e não outra pessoa que terá a responsabilidade pertinente.

É freqüente a proposição de uma série de recomendações ao doente, sem que se leve em conta a complexidade dos procedimentos, capacidade de sua aplicação, custo e possíveis reações adversas ao medicamento. Conseqüentemente, é comum o baixo nível de aderência, particularmente, quando se exigem procedimentos de tratamento ou emprego contínuo de medicamento durante período prolongado. É o que se observa em diversas condições e doenças, como, por exemplo, hemodiálise, hipertensão arterial, epilepsia, tuberculose, diabetes, artrite reumatóide, esquizofrenia, depressão maior e transtorno afetivo bipolar.

De maneira geral, a taxa de aderência é em torno de $50 \%{ }^{(17)}$. Essa porcentagem torna-se menor nos tratamentos longos ou profiláticos, nas condições clínicas discretas ou assintomáticas e nas doenças cujas conseqüências da interrupção demoram para surgir. Nos pacientes psiquiátricos a aderência tende a ser menor ${ }^{(17-}$ 19).

Fatores sociodemográficos - idade, sexo, raça, inteligência e educação - não têm correlação com a aderência $^{(20)}$.

A cultura de um povo ou país pode influenciar no seguimento das recomendações. A não-aderência é mais comum em pacientes submetidos à hemodiálise nos Estados Unidos da América do Norte (EUA) do que no Japão e na Suécia ${ }^{(21)}$.

Doentes tendem a faltar nas consultas ou a abandonar o tratamento quando necessitam esperar muito tempo para serem atendidos nas clínicas ou quando é longo o intervalo entre as consultas ${ }^{(20)}$.

A falha na aderência ao tratamento pode abranger a não aquisição da prescrição, recusa em tomar o medicamento, administração irregular ou em quantidade insuficiente, dificuldade com os diversos horários e seqüência dos vários medicamentos, e, finalmente, a própria interrupção do tratamento ${ }^{(22)}$. Já no início da década de 60, quando os antipsicóticos passaram a ser utilizados em maior escala e como recurso imprescindível no tratamento da esquizofrenia, foi demonstrado que $48 \%$ dos doentes acompanhados em ambulatório não tomavam o medicamento ${ }^{(23)}$. Outro estudo apontou que $20 \%$ dos pacientes hospitalizados, sob controle supostamente rigorosos, falhavam em tomar toda a medicação prescrita $^{(24)}$. Passados 40 anos, o problema ainda persiste e em porcentagem equivalente. Na década de 90 , uma análise indicou que, a taxa de aderência foi de $40 \%$ no primeiro ano após a alta hospitalar ${ }^{(25)}$. Mesmo com todo o progresso que temos acompanhado, com o advento dos antipsicóticos de nova geração, apresentando perfil de 
efeitos colaterais mais favorável, melhorando a tolerabilidade e aceitação, permanece a dificuldade da aderência $^{(26)}$.

\section{FATORES QUE PREJUDICAM A ADERÊNCIA AO TRATAMENTO DE MANUTENÇÃO}

Os seguintes fatores podem ser considerados: paciente, medicamento, meio ambiente e aliança terapêutica. Serão analisados separadamente, embora estejam intimamente interligados.

No que se refere ao paciente, vários aspectos são observados, por exemplo, as condições intrínseicas à própria psicopatologia (desorganização, idéias delirantes - principalmente de cunho paranóide - hostilidade, déficit cognitivo, falta de motivação e de iniciativa) e a gravidade da doença. A falta de insight, ou seja, a dificuldade em compreender e reconhecer os sintomas e a doença, induz muitos pacientes a considerarem que não necessitam de tratamento. Ao mesmo tempo, determinado número deles não conseguem estabelecer a associação entre interrupção da medicação e recaída. Outros, ao se recuperarem adequadamente, não reconhecem os efeitos benéficos do medicamento, levam em conta o custo do tratamento (efeitos adversos, estigma e aspectos econômicos), o quanto de fato estão doentes e se têm, realmente, necessidade de tratamento. Na seqüência, manifestam o desejo de permanecer sem medicamento. Porcentagem determinada de doentes refere não tomar o medicamento devido ao esquecimento ou porque deseja de outro tipo de tratamento. A dificuldade em estabelecer aliança terapêutica contribui para irregularidade do comparecimento à consultas e do seguimento da conduta. Os pacientes que fazem uso de álcool e drogas são mais propensos a não tomar o medicamento ${ }^{(5,24,27-31)}$.

Nos fatores relacionados ao medicamento, incluem-se a complexidade do regime de tratamento e a ocorrência de efeitos colaterais. Os desconfortos subjetivos (disforia e embotamento cognitivo) e físicos (sedação, acinesia, aumento do peso e disfunção sexual) interferem na qualidade de vida e ocasionam interrupção do tratamento ${ }^{(29,32)}$.

Quanto ao meio ambiente, a participação de membros da família e amigos no programa de assistência favorece a aderência do paciente no tratamento, auxilia no controle ou ajuste de fatores psicossociais, na compreensão sobre a doença e a necessidade de cuidados pertinentes. O mesmo se pode dizer quanto ao apoio social e estrutura da comunidade, por exemplo, fornecimento de medicamento, de transporte facilitando o deslocamento para os locais de atendimento e programas de atividades em centros comunitários ou hospital-dia ${ }^{(22,29-30)}$. Ao lado do medicamento, a implementação de recursos relacionados a este fator é de fundamental importância na reabilitação do paciente.

Outro fator essencial é a aliança terapêutica. Por este termo entende-se a qualidade de cooperação e entendimento que se estabelece entre o paciente e 0 terapeuta. Um bom relacionamento proporciona ambiente seguro e confiável; envolve, integra e mobiliza o cliente no tratamento. Atualmente, já se encontra bem estabelecido que o fortalecimento da aliança terapêutica é de fundamental importância para a regularidade do seguimento e na tomada do medicamento ${ }^{(30,33)}$. A prescrição do medicamento sem estabelecer relacionamento adequado e sem explicar o que se espera, está fadada ao insucesso ${ }^{(8)}$. O psiquiatra e equipe multidisciplinar devem estar cientes de que a esquizofrenia exige tratamento prolongado e é, muitas vezes, de resultado limitado. Mesmo sabendo da dificuldade que esses pacientes exibem para estabelecer a aliança terapêutica ${ }^{(33)}$, é necessário ter apreço do doente, aceitá-lo e desejar tratá$1 \mathrm{o}^{(8)}, \mathrm{e}$, acrescentamos, ter o espírito humano e dedicação genuína a este tipo de assistência.

\section{CONSEQÜÊNCIAS DA NÃO-ADERÊNCIA AO TRATAMENTO}

Em 1911, Bleuler descrevia a esquizofrenia como uma doença crônica, caracterizada por fases de exacerbação que podiam deter-se ou retroceder-se em qualquer momento, mas, sem uma completa restitutio ad integrum.

O tratamento adequado com antipsicótico, por ocasião do primeiro episódio possibilita a obtenção de remissão completa em $74 \%$ dos casos e parcial, em $12 \%{ }^{(34)}$. Entretanto, no transcorrer da doença e do tratamento de manutenção, o nível de resposta em geral se modifica - cerca de $25 \%$ dos pacientes mantém a melhora acentuada, e $50 \%$ passam a apresentar melhora parcial e 25\% não responde ao tratamento ou apresenta piora da sintomatologia ${ }^{(35-36)}$. Observa-se que é elevado o número de doentes com remissão incompleta, com persistência da incapacidade. 
O grande risco da não-aderência é o agravamento da doença. Defrontando-se com esta condição, o clínico deve fazer o diagnóstico diferencial e avaliar os possíveis fatores que estariam envolvidos, por exemplo, curso natural da doença, subdosagem, uso irregular do medicamento, interrupção do tratamento, situações estressoras ou uso de drogas ${ }^{(37)}$.

A não-aderência tende a se desenvolver gradualmente em muitos pacientes e suas conseqüências, o agravamento da sintomatologia, não serem notadas de imediato. Alguns meses poderão se passar, em média de três a sete meses, até que começa a surgir, insidiosamente ou repentinamente, a recaída ${ }^{\text {(38- }}$ ${ }^{39)}$. Nas condições de determinado estudo, pacientes com apenas um episódio e estabilizados com medicamento há um ano, o tempo médio para o desencadeamento da recorrência, após a interrupção da medicação, foi de aproximadamente oito meses ${ }^{(31)}$.

A taxa média de recaída em pacientes submetidos a placebo durante um ano pode variar de 30 a $86 \%$. Essa variação depende da população estudada e do critério de definição de recaída adotado: será maior quando baseado no reaparecimento dos sintomas e, menor, em reinternação ${ }^{(38)}$.

Outra investigação analisou a taxa de recaída em pacientes que acabaram de ter o primeiro episódio de esquizofrenia e permaneceram estáveis durante um ano de tratamento. Seguindo determinado protocolo de estudo, ao se interromper o medicamento, a porcentagem de pacientes que tiveram exacerbação discreta ou grave dos sintomas psicóticos no período de um ano foi de $78 \%$. Em dois anos, $96 \%{ }^{(31)}$.

A maioria dos pacientes apresenta agravamento da psicopatologia no decorrer de cinco anos e esse risco aumenta, em três a cinco vezes, quando deixa-se de tomar a medicação antipsicótica ${ }^{(40)}$.

As conseqüências da recaída são diversas, variando desde a ruptura psicossocial, utilização de serviços de emergência, necessidade de internação ou de maior número de consultas para o controle clínico, aumento do custo financeiro para os serviços públicos e da própria família, desajuste do ambiente familiar estresse devido ao estado do paciente, que atinge, algumas vezes, comportamento agressivo ou hostil, necessidade de algum membro da família faltar ao trabalho para tomar conta do doente. Em determinados casos, em conseqüência às recaídas, o tempo para recuperação torna-se mais longo e a resposta inferior ao tratamento.
Levando-se em conta estes dados, nos casos com remissão parcial, associados ou não a múltiplos episódios, recomenda-se a manutenção do antipsicótico por tempo indeterminado. O objetivo é a prevenção da exacerbação e agravamento da psicopatologia.

\section{IMPLICAÇÕES CLÍNICAS E BENEFÍCIOS DOS ANTIPSICÓTICOS DE AÇÃO PROLONGADA}

Em primeiro lugar, convém destacar a certeza da administração do medicamento e da dose prescrita, quando aplicados por via intramuscular. Ao mesmo tempo, a garantia da regularidade e continuidade do tratamento, condições essenciais na prevenção de recaída. Os pacientes com maior probabilidade de se beneficiar com estes medicamentos são aqueles que não cooperam com o tratamento ${ }^{(37,41)}$. É possível, desta forma, e desde que esteja sendo administrada a dose eficaz da medicação, a redução das recaídas e internações, permitindo que maior número de doentes seja mantido em tratamento ambulatorial e no ambiente familiar e social.

O resultado de estudos a duplo-cego indica redução da taxa de recaída e de re-hospitalização em pelo menos $10 \%$ dos casos $^{(37)}$, com benefício evidente às famílias e aos serviços de saúde pública ${ }^{(12)}$.

O esquema posológico é simplificado: dose única, v.o., uma vez por semana, no caso do penfluridol (semap) ou i.m., uma vez a cada 7 a 30 dias, para os demais. Este regime contribui para a aderência ao tratamento, melhora do ambiente familiar, reduzindo atritos e tensões para que o paciente tome o medicamento. Em sua experiência de 12 anos com antipsicóticos de ação prolongada, Freeman ${ }^{(42)}$ chamara atenção para a maior participação e integração ao tratamento dos doentes com este tipo de medicamento. Numa revisão sistemática realizada, os pacientes, de forma geral, indicavam a aceitação e preferência pela medicação depot em relação à oral ${ }^{(43)}$. Neste contexto, explicando esta opção, deparamo-nos com pacientes que referem o seguinte: "a cada dia em que pego o comprimido e o levo à boca, lembro que sou um psicótico! Prefiro a injeção a cada 15 a 30 dias. Diminui minha sensação de ser doente". Ao lado destes dados favoráveis, deve-se destacar que o preparo e a educação do doente e da família, antes de se administrar o medicamento e no decurso do tratamento, são essenciais para evitar o risco de interrupção ${ }^{(5)}$. 
A vantagem farmacológica inclui ausência do metabolismo de primeira passagem. Com os antipsicóticos de ação curta, administrados v.o., uma parte substancial do medicamento é metabolizada no fígado antes de alcançar a circulação sistêmica. Os de ação prolongada são absorvidos pela corrente sangüínea, passam pelos pulmões e chegam ao cérebro, sem terem sofrido metabolização. Quanto à farmacocinética, os depot propiciam concentração plasmática mais estável e constante do que os de ação curta.

Se o paciente falhar ou atrasar uma das aplicações, não ocorre interrupção abrupta do medicamento, sendo pouco provável predispor a recaída ${ }^{(37)}$. Em muitos casos, após interromper a medicação depot, o nível plasmático ainda se mantém em proporção substancial após três meses ${ }^{(31)}$.

As facilidades descritas evitam desperdício de medicamentos, que freqüentemente são acumulados, escondidos ou desprezados pelos doentes que se recusam a tomá-los.

Para os casos bem controlados, em que existe colaboração adequada da família e do doente para a aplicação em domicílio, a ação prolongada do antipsicótico permite que as consultas possam ser espaçadas para um período de até quatro meses, resultando nos seguintes benefícios: 1. redução de despesas com consulta, transporte e refeição para os que residem em outras cidades, vizinhas ou distantes; 2. economia de tempo, para os que se reintegraram no trabalho ou para os acompanhantes, reduzindo-se a necessidade de justificativa das ausências; 3 . diminuição da opressão ou estigma de ser doente.

Do ponto de vista de saúde pública há vantagem considerável em poder contar com medicamento que é administrado em intervalo de 15 a 30 dias: simplifica-se a administração terapêutica e aumenta a garantia da regularidade do tratamento, torna mais econômica a vigilância sanitária, com a redução das recaídas e internações, sem prejuízo dos benefícios ao doente, à família e à comunidade.

\section{ACEITAÇ̃̃O E PRESCRIÇÃO DOS ANTIPSICÓTICOS DE AÇÃO PROLONGADA}

Embora o efeito terapêutico esteja comprovado e os benefícios sejam evidentes, o emprego destes medicamentos nos diversos países está relacionado com a tendência psiquiátrica e cultural de cada um deles ${ }^{(13)}$. Dados recentes indicam que ocorre variação inclusive dentro de um mesmo país ou continente, como será visto a seguir.

Cita-se que, nos Estados Unidos da América do Norte, os antipsicóticos de ação prolongada são utilizados em $5 \%$ a $20 \%$ dos pacientes ${ }^{(39)}$. No levantamento realizado em 14 centros (Veterans Administration Medical Centers), foi verificado que $17,90 \%$ dos doentes receberam antipsicótico depot, com variação entre $2 \%$ a $28 \%$ de acordo com a localidade ${ }^{(32)}$.

Estudo realizado em 10 países da Europa, entre 1 ㅇ de setembro de 2000 e 31 de dezembro de 2001, indica que $24,1 \%$ dos esquizofrênicos receberam antipsicótico de ação prolongada. O seu emprego não é uniforme: $45,6 \%$ em Portugal, $45,5 \%$ na Irlanda, 38\% no Reino Unido, $28,6 \%$ na Itália, 22,3\% na Espanha, 22,6\% na França, $22,2 \%$ na Alemanha, $18,1 \%$ na Holanda, $15,2 \%$ na Dinamarca e 10,7\% na Grécia ${ }^{(44)}$.

$\mathrm{Na}$ Austrália, durante o segundo semestre de 2001, a porcentagem dos depot aviados nas farmácias, em relação aos demais antipsicóticos, foi de $16,7 \%{ }^{(45)}$. No Brasil, em 1998, o decanoato de haloperidol, o palmitato de pipotiazina e o penfluridol corresponderam aproximadamente a $5 \%$ do total das prescrições em nossas farmácias ${ }^{(46)}$.

$\mathrm{Na}$ década de 70, vários autores manifestaramse sobre o caráter coercivo dos antipsicóticos depot. Consideravam que o doente "perdia sua liberdade", ficava "dominado à distância" e submetido à "internação" em seu próprio domicílio. Contudo, deve-se ponderar que o episódio psicótico traduz alteração mental, caracterizado por percepção distorcida, lógica defeituosa e juízo crítico diminuído. Nessas condições, o indivíduo encontra-se em situação precária para proteger-se e enfrentar os obstáculos do dia-a-dia. Além disto, o distúrbio psicótico causa sofrimento, sendo que $25 \%$ a $50 \%$ dos esquizofrênicos tentam suicídio e $4 \%$ a $13 \%$, cometem suicídio $^{(47)}$. Cabe à família conduzir o doente ao médico e a este estabelecer o plano de tratamento.

Em nosso meio, durante muitos anos, contávamos apenas com o enantato de flufenazina (anatensol depot). Desde o início foi possível observar sua predisposição em desencadear intensas reações 
extrapiramidais, desestimulando o seu emprego. Por esta razão, nos EUA, este medicamento foi logo substituído pelo decanoato de flufenazina. Entretanto, no Brasil, o enantato de flufenazina continuou sendo utilizado, particularmente, em serviços públicos, mesmo após a introdução de outros. Em 1982 e 1985, passamos a contar, na seqüência, com o palmitato de pipotiazina e decanoato de haloperidol. Todavia, permaneceu a imagem negativa da associação "antipsicóticos depot e risco de efeitos colaterais". Muitos psiquiatras acabaram não os considerando como melhor opção de tratamento e transferindo, de alguma forma, esta noção aos pacientes e familiares. Outro aspecto que, possivelmente, possa ter restringido os psiquiatras a aderir ao emprego dos depot é a necessidade da administração i.m. Alguns psiquiatras sentem certo desconforto em aplicar injeções ${ }^{(48)}$. Outros, não manifestam a intenção de mudar a rotina de trabalho. Há, ainda, aqueles que argumentam que este procedimento afeta, de alguma forma, o relacionamento médico-paciente. Em conseqüência disso, este importante recurso tem sido pouco aproveitado.

$O$ tratamento da esquizofrenia com os antipsicóticos de ação prolongada requer metodologia. Não basta apenas fazer a prescrição. É necessário dar todo suporte ao paciente e à família. Entre os diversos procedimentos, está o controle das datas de consultas e das aplicações i.m. Em consultório particular, estabelecemos que, devido a eventual não comparecimento, contato por telefone será realizado fixando nova data. A administração i.m., efetuada pelo próprio médico, garante a certeza do tratamento e da regularidade. A aplicação destas medidas e de outros cuidados durante o tratamento implica num estilo de assistência médica a ser adotado. Convencionalmente, o psiquiatra entrega a prescrição ao paciente e à família, que por sua vez passam a responsabilidade à outra pessoa que não está inserida no programa de assistência. Neste sistema de atendimento, perde-se o controle do tratamento profilático. Mudanças são necessárias para que se coloque em prática clínicas de antipsicóticos de ação prolongada, tanto em consultório particular quanto em serviço ambulatorial público, adotando-se estratégias apropriadas às condições da doença.

Os antipsicóticos de ação prolongada constituem recurso apreciável a ser utilizado no tratamento de manutenção, independentemente do paciente aderir ou não à proposta terapêutica. De nossa parte consideramos, entre as diversas vantagens, a praticidade. É inegável esta condição. Mesmo para o doente que adere ao tratamento, tomar um comprimido por semana é mais simples do que todos os dias. Assim é válido para qualquer regime medicamentoso. O penfluridol ( semap) enquadra-se nesta condição. Por outro lado, os antipsicóticos de ação prolongada i.m., asseguram o tratamento, não importando o grau de cooperação. Este recurso simplifica e garante!

O conceito que os psiquiatras e participantes de equipes de saúde mental possuem a respeito dos antipsicóticos de ação prolongada determina o seu emprego em maior ou menor escala. Pelos benefícios inestimáveis que eles podem oferecer, uma campanha a nível nacional, sem dúvida, muito contribuiria para melhor utilização deste recurso apreciável no tratamento da esquizofrenia.

Nos últimos dez anos, o enfoque do tratamento da esquizofrenia tem se concentrado nos antipsicóticos de nova geração. Pelas vantagens em relação aos convencionais $^{(46)}$, houve um aumento apreciável de suas prescrições e, conseqüentemente, um declínio dos demais, inclusive dos depot. Entretanto, surge agora um agente de nova geração e de ação prolongada, o Risperdal Consta $^{(11)}$. Este aprimoramento da terapêutica abre nova perspectiva no tratamento da esquizofrenia.

\section{CONCLUSÃO}

A taxa de não aderência ao tratamento na esquizofrenia é em torno de $60 \%$. Por se tratar de uma doença crônica com elevado risco de recaída, a manutenção do medicamento auxilia no seu controle. Os antipsicóticos de ação prolongada foram desenvolvidos exatamente com este objetivo: garantir a administração do medicamento e a sua regularidade, condições essenciais na prevenção da recaída.

Apesar dos benefícios inestimáveis dos antipsicóticos de ação prolongada, diversos fatores contribuíram para que fossem utilizados aquém do seu potencial. 


\section{REFERÊNCIAS BIBLIOGRÁFICAS}

1. Bechelli LPC, Hetem G. Neurolépticos de ação propongada (N.A.P.). Ação, indicação e efeitos colaterais. Rev Bras Medicina 1977; 34:667-76.

2. Bechelli LPC, Ruffino-Netto A, Hetem G, Scaloppi EL. Le palmitate de pipotiazine (19552 RP) neuroleptique à action prolongée, administré mensuellement comme traitement d'entretien ambulatoire de sujets atteints de schizophrenie. Actualités Psychiatriques 1979; 8:134-7.

3. Bechelli LPC, lecco MC, Aciolo A, Pontes MC. A doubleblind trial of haloperidol decanoate and pipothizine palmitate in the maintenance treatment of schizophrenics in a public out-patient clinic. Curr Ther Res 1985; 37:662-71.

4. Bechelli LPC, Hetem G. Long-acting pipotiazine administered in monthly doses for the maintenance treatment of schizophrenic patients in a private practice. Curr Ther Res 1981; 29:903-14.

5. Bechelli LPC, Hetem G. Pipotiazina de ação prolongada e de administração mensal no tratamento de manutenção de esquizofrênicos em consultório particular com seguimento máximo de cinco anos. J Bras Psiquiatr 1984; 33:451-6.

6. Bechelli LPC. Neuroléptico de ação prolongada (NAP) na fase aguda da esquizifrenia. Rev Assoc Bras Psiq 1982; 4:20810.

7. Bechelli LPC, Navas F Filho. Short-term double-blind trial of pipothiazine palmitate and haloperidol in the acute phase of schizophrenia. L'Encephale 1986; 12:121-5.

8. Bechelli LPC, Hetem G. Neurolépticos e Psicoterapia. J Bras Psiquiatr 1983; 32:409-13.

9. Bechelli LPC. Neurolépticos de ação prolongada (NAP) no tratamento ambulatorial de esquizofrênicos: preparo da alta hospitalar e aspectos sócio-econômicos. J Bras Psiquiatr 1984; 33:56-9.

10. Bechelli LPC. Neurolépticos de Ação Prolongada. In: Caetano D, Frota-Pessoa O, Bechelli LPC, organizadores. Esquizofrenia. Atualização em Diagnóstico e Tratamento. São Paulo (SP): Atheneu; 1993. p. 187-95.

11. Kane JM, Eerdekens M, Lindenamyer J-P, Keith SJ, Lesem $\mathrm{M}$, Karcher K. Long-acting injectable risperidone: efficacy and safety of the first-long-acting atypical antipsychotic. Am J Psychiatry 2003; 160:1125-32.

12. Bechelli LPC. Neurolépticos - Efeitos terapêuticos e colaterais em doentes de esquizofrenia. Impacto nos serviços de saúde mental. J Bras Psiquiatr 1986; 35:3-9.

13. Bechelli LPC. Preparações depot em doentes esquizofrênicos em consultório particular e em ambulatório público. J Bras Psiquiatr 1982; 31:143-7.

14. Burke JC, High JP, Laffan RJ, Ravaris CL. Depot action of fluphenazine (prolixin) enanthate in oil. Fed Proc 1962; 21:339. 15. Kinross-Wright J, Vogt AH, Charalampous KD. A new method of drug therapy. Am J Psychiatry 1963; 119:779-80.

16. Bechelli LPC. Antipsicóticos de Ação Prolongada no Tratamento de Manutenção da Esquizofrenia. Parte II. O manejo do medicamento e integração dos serviços médico, social, de enfermagem e de psicologia. Rev Latino-am Enfermagem 2003; 11(4). No prelo.

17. Haynes RB, McDonald H, Garg AX, Montague P. Interventions for helping patients to follow prescriptions for medications (Cochrane Review). In: The Cochrane Library, Issue 1. Oxford: Update Software. 2003.

18. Cramer JA, Rosenheck R. Compliance with medication regimens for mental and physical disorders. Psychiatr Serv 1998; 49:196-201.
19. Scherer ZAP, Scherer EA. O doente mental crônico internado: uma revisão da literatura. Rev Latino-am Enfermagem 2001; 9(4):56-61.

20. McDonald HP, Garg AX, Haynes RB. Intervations to enhance patient adherence to medication prescription. JAMA 2002; 288:2868-79.

21. Bleyer AJ, Hylander B, Sudo H, Nomoto $Y$, de la Torre E, Chen RA, Burkart JM. An international study of patient compliance with hemodialysis. JAMA 1999; 281:1211-3.

22. Perkins DO. Predictors of noncompliance in patients with schizophrenia. J Clin Psychiatry 2002; 63:1121-8.

23. Wilcox DCR, Gillan R, Hare EH. Do psychiatric out-patients take their drugs? Br Med J 1965; 2:790-2.

24. Kline NS, Simpson GM. A long-acting fluphenazine in office practice. Am J Psychiatry 1964; 120:1012-1-14.

25. Weiden $P$, Olfson $M$. The cost of relapse in schizophrenia. Schizophr Bull 1995; 21:419-28.

26. Dolder CR, Lacro JP, Dunn LB, Jeste DL. Antipsychotic medication adherence: is there a difference between typical and atypical agents? Am J Psychiatry 2002; 159:103-8.

27. Haywood TW, Kravitz HM, Grossman LS, Cananaugh JL, Davis JM, Lewis DA. Predicting the "revolving door" phenomenon among patients with schizophrenic, schizoaffective, and affective disorders. Am J Psychiatry 1995; 152:856-61.

28. Owen RR, Fisher EP, Booth BM, Cuffel BJ. Medication noncompliance and substance abuse among patients with schizophrenia. Psychiatr Serv 1996; 47:853-8.

29. Fenton WS, Blyler CR, Heinssen RK. Determinants of medication compliance in schizophrenia: empirical and clinical findings. Schizophr Bull 1997; 23:637-51.

30. Olfson M, Mechanic D, Hansell S, Boyer CA, Walkup J, Weiden PJ. Predicting medication noncompliance after hospital discharge among patients with schizophrenia. Psychiatr Serv 2000; 51:216-22.

31. Gitlin M, Nuechterlein K, Subotnick KL, Ventura J, Mintz J, Fogelson DL, Bartzokis G, Aravagiri M. Clinical outcome following neuroleptic discontinuation in patients with remitted recent-onset schizophrenia. Am J Psychiatry 2001; 158:183542.

32. Valenstein M, Copeland LA, Owen R, Blow FC, Visnic S. Adherence assessment and the use of depot antipsychotics in patients with schizophrenia. J Clin Psychiatry 2001; 62:54551.

33. Frank AF, Gunderson JG. The role of the therapeutic alliance in the treatment of schizophrenia: relationship to course and outcome. Arch Gen Psychiatry 1990; 47:228-36. 34. Lieberman JA, Jody D, Geisler S, Alvir J, Loebel A, Szymanskis S, Woerner M, Borenstein M. Time course and biological correlates of treatment response in first-episode schizophrenia. Arch Gen Psychiatry 1993; 50:369-76.

35. Cutting J. Outcome of schizophrenia: overview. In: Kerr TA, Snaith RP, organizers. Contemporary issues in schizophrenia. London: Royal College of Psychiatrist; 1986. p. 433-40.

36. Louzã Neto MR. Esquizofrenia. In: Louzã Neto MR, Motta T, Wang Y-P, Elkis H, organizadores. Psiquiatria Básica. Porto Alegre: Artes Médicas; 1995. p. 167-204.

37. Kane JM, Aguglia E, Altamura AC, Gutierrez JLA, Brunello N, Fleichhacker WW, Gaebel W, Gerlach J, Guelfi J-D, Kissling W, Lapierre YD, Lindström, Mendlewicz J, Racagni G, Carulla LS, Schooler NR. Guidelines for depot antipsychotic treatment in schizophrenia. Eur Neuropharmacol 1998; 8:55-66.

38. Kane JM, Lieberman JA. Maintenance pharmacotherapy in schizophrenia. In: Meltzer HY, organizador. Psychopharmacology: The Third Generation of Progress. New York: Raven Press; 1987. p. 1103-9. 
39. Glazer WM, Kane JM. Depot neuroleptic therapy: an underutilized treatment option. J Clin Psychiatry 1992; 53:42633.

40. Robinson D, Woener MG, Alvir JMJ, Bilder R, Goldman R, Geisler S, Koreen A, Sheitman B, Chakos M, Mayerhoff D, Lieberman JA. Predictors of relapses following response from a first episode of schizophrenia or schizoaffective disorder. Arch Gen Psychiatry 1999; 56:241-7.

41. Davis JM, Metalon L, Watanabe MD, Blake L. Depot antipsychotic drugs. Place in therapy. Drugs 1994; 47:74173.

42. Freeman H. Twelve years' experience with total use of depot neuroleptic in a defined population. In: Cattabeni $F$, Racagni G, Spano PF, Costa E, organizadores. Long-term effects of neuroleptics (Adv. Biochem. Psychopharmacol Vol. 24). New York: Raven Press; 1980. p. 559-64.

43. Walburn J, Gray R, Gournay K, Quraishi S, David AS. Systematic review of patient and nurse attitudes to depot antipsychotic medication. Br J Psychiatry 2001; 179:300-7. 44. Haro JM, Edgell ET, Frewer P, Alonso J, Jones PB on behalf of the SOHO Study Group. The European Schizophrenia Outpatient Health Outcome Study: baseline findings across country and treatment. Acta Psychiatr Scand 2003; 107 (Suppl. 416):7-15.

45. Mond J, Morice R, Owen C, Korten A. Use of antipsychotic medication in Australia between July 1995 and December 2001. Australian and New Zealand Journal of Psychiatry 2003; 37:55-61.

46. Bechelli LPC. Impacto e evolução do emprego dos neurolépticos no tratamento da esquizofrenia. J Bras Psiquiatr 2000; 49:131-47.

47. Meltzer HY. Treatment of suicidality in schizophrenia. Annals of the New York Academy of Sciences 2001; 932:4460.

48. Balon R. The underutilization of depot neuroleptic therapy (letter). J Clin Psychiatry 1994; 55:39. 